[7] Uchida et al. Asian J. Endosc. Surg. 2018

[8] Hart et al. Mod. Pathol. Off. J. U. S. Can. Acad. Pathol. Inc 2015

[9] Kim et al. Tuberc. Respir. Dis. 2014

Disclosure of Interests: Jan Schirmer: None declared, Marcus Seeger: None declared, Dominik Schulte Paid instructor for: Ferring, Speakers bureau: Amgen, Astra Zeneca, Braun, Fresenius, Ipsen, Johnson \& Johnson, Lilly, MSD, Novartis, Novo Nordisk, Sanofi, Matthias Laudes Speakers bureau: Roche, Sanofi, Chugai, Stefan Schreiber Consultant of: AbbVie, Arena, BMS, Biogen, Celltrion, Celgene, IMAB, Gilead, MSD, Mylan, Pfizer, Fresenius, Janssen, Takeda, Theravance, provention Bio, Protagonist and Falk, Bimba F. Hoyer: None declared

DOI: 10.1136/annrheumdis-2020-eular.3181

\section{THU0604 UNDIAGNOSED RHEUMATIC DISEASE IN NEWLY PRESENTING MGUS PATIENT}

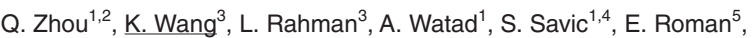
D. Mcgonagle ${ }^{1} .{ }^{1}$ National Institute for Health Research, Leeds Biomedical Research Centre and Leeds Institute of Rheumatic and Musculoskeletal Medicine (LIRMM), Leeds, United Kingdom; ${ }^{2}$ Sichuan Academy of Medical Sciences \& Sichuan Provincial People's Hospital, Department of Rheumatology and Immunology, Chengdu, China; ${ }^{3}$ University of Leeds, School of Medicine, Leeds, United Kingdom; ${ }^{4}$ St James's University Hospital, Department of Clinical Immunology and Allergy, Leeds, United Kingdom; ${ }^{5}$ University of York, Epidemiology \& Cancer Statistics Group (ECSG), Department of Health Sciences, York, United Kingdom

Background: Monoclonal gammopathy of undetermined significance (MGUS) is considered to be a premalignant condition with on average of $1 \%$ annual risk of progression to multiple myeloma or other lymphoproliferative disorders [1]. Numerous studies have highlighted a common feature of autoimmune inflammatory diseases is non-specific hypergammaglobulinaemia which can be associated with monoclonal gammopathy [2, 3]. We looked at a population of 3.6 million where patients with MGUS was referred to haematology network for evaluation.

Objectives: Our hypothesis was that undiagnosed rheumatic diseases were being referred to haematology rather than rheumatology erroneously.

Methods: The Haematological Malignancy Research Network (HMRN) ethics approval (REC 04/01/1205/69) from Leeds West Research Ethics Committee. The HMRN that comprises a population-based cohort of patients newly diagnosed by a single integrated haemato-pathology laboratory in two adjacent UK Cancer Networks (population 3.6 million). The database includes prognostic factors, sequential treatment/response history and socio-demographic details which are recorded to clinical trial standards. 255 patients were screened in this study. We looked at a range of autoimmune/innate immune conditions diagnosed after MGUS.

Results: In the 255 patients cohort group, the average age at the diagnosis of MGUS was $70.23 \pm 11.95$ years (median 70.2 years), with more subjects being male $(n=145,56.9 \%)$. Mean duration of follow up was 2570 days. 10 out of the 255 patients progressed onto multiple myeloma.

Diagnosed rheumatic disease was found in 48 patients (18.8\%). None of the patient in this group has disease progression to multiple myeloma. In this group, 37 patients (14.5\%) presented the rheumatic disease before their MGUS diagnosis and $11(4.3 \%)$ were diagnosed after their MGUS referral. Interestingly, among the 11 , more males $(n=8,72.7 \%)$ have their rheumatic disease diagnosed after MGUS.

Those 11 cases included crohn's disease (1), polymyalgia rheumatica (2), immune thrombocytopenia (2), autoimmune hepatitis (2), Schnitzler's syndrome (1), giant cell arteritis (1), rheumatoid arthritis (2).

Conclusion: Approaching 1 in 20 cases of MGUS have an underlying inflammatory disease that may often be non-specifically driving antibody production including monoclonal band formation. When diagnosing MGUS, clinicians should be aware of the potential underlying autoimmune rheumatic conditions.

References:

[1] Kyle, R.A., et al., A long-term study of prognosis in monoclonal gammopathy of undetermined significance. N Engl J Med, 2002. 346(8): p. 564-9.

[2] Renier, G., et al., Ankylosing spondylitis and monoclonal gammopathies. Ann Rheum Dis, 1992. 51(8): p. 951-4.

[3] Sugai, S., et al., Non-IgM monoclonal gammopathy in patients with Sjogren's syndrome. Am J Med, 1980. 68(6): p. 861-6.

Disclosure of Interests: Qiao Zhou: None declared, Kaiwen Wang: None declared, Laiba Rahman: None declared, Abdulla Watad: None declared, Sinisa Savic: None declared, Eve Roman: None declared, Dennis McGonagle Grant/ research support from: Janssen Research \& Development, LLC

DOI: 10.1136/annrheumdis-2020-eular.4320

\section{THU0605 AN ATYPICAL CASE OF PONCET DISEASE}

M. Yasmine ${ }^{1}$, H. Ajlani ${ }^{1}$, S. Boussaid ${ }^{1}$, S. Jammali ${ }^{1}$, S. Rekik ${ }^{1}$, E. Choeur ${ }^{1}$, H. Sahli Srairi ${ }^{1}$, M. El Euch ${ }^{1}{ }^{1}$ Rabta Hospital, Tunis, Tunisia

Background: Poncet disease (PD) is defined as an inflammatory rheumatism associated with visceral tuberculosis without direct bacteriological involvement of the joints [1]. It is classified as a parainfectious rather than a reactive arthritis [2]

Objectives: Here by a first case of PD who presented with sterile arthritis and tuberculous spondylodiscitis.

Methods: We report a case of a 40-year-old women who presented with polyarthritis in 2014. On physical examination, she had synovitis in both wrists, the metacarpophalangeal joints and the fifth proximal interphalangeal joint of the right hand. Her serum was negative for Rheumatoid Factor (RF) and anti-cyclic citrullinated peptide (anti-CCP) antibody. Her C-reactive protein (CRP) was $24,5 \mathrm{mg} / \mathrm{l}$. Ultrasound revealed tenosynovitis of the superficial and deep flexor tendons on both hands with Doppler signal. The Magnetic resonance imaging (MRI) of the hands showed active synovitis in the wrists mainly in the distal radioulnar joint, erosions in the ulnar styloid as well as edematous infiltration of the soft tissue of the hands. Since she fulfilled the new ACR/EULAR 2010 criteria for RA, a diagnosis of rheumatoid arthritis (RA) was made and the patient was put on Methotrexate (MTX) $15 \mathrm{mg} /$ week/po in January 2015. Eight months later, the patient developed high temperature $38^{\circ} \mathrm{C}$ and lumbar stiffness. A chest CT performed as part of the etiologic investigation didn't show pulmonary manifestations but revealed a lytic vertebrae lesion. Lumbar spine MRI showed prevertebral edema and soft tissue enhancement with abnormal marrow signal in L2 and L3 which was concerning for infectious etiology. MTX was stopped. A CT-guided core needle biopsy concluded to a tuberculous spondylodiscitis. The patient was initiated on an antituberculous-therapy (ATT) for 15 months. The course was marked by the reoccurrence of low back pain. MRI of the spine was then performed and revealed persistence of spondylodiscitis and multiple abscesses at the levels of L2-L3. The ATT was resumed.

Results: The patient received four drugs for 4 months, followed by isoniazid and rifampicin for 1 year. At follow up, the patient responded well to treatment with complete resolution of symptoms without sequelae. She did not present neither polyarthritis nor synovitis. Moreover, she sustained a negative CRP (2mg/dl). Ultrasound control of the wrists did not show synovitis or tenosynovitis Doppler signal. Similarly, a disappearance of effusion as well as synovitis was noted on the MRI at follow up.

Conclusion: We report a unique case of Poncet disease with tubercular spondylodiscitis. It is important to recognize PD in a patient presenting with polyarthritis in order to avoid unnecessary long-term disease-modifying antirheumatic treatment. Future research is indicated to understand the etiopathogenesis of Poncet's disease and to educate clinicians as to the importance of maintaining a high index of suspicion about this rare, yet potentially easily treatable disease.

References:

[1] Sood R, Wali JP, Handa R. Poncet's disease in a north Indian hospital. Trop Doctor. 1999; 29: 33-6.

[2] Bloxham CA, Addy DP. Poncet's disease: parainfective tuberculous polyarthropathy. Br Med J. 1978; 1: 1590.

Disclosure of Interests: None declared

DOI: 10.1136/annrheumdis-2020-eular.3512

\section{\begin{tabular}{|l|l}
\hline THU0606 & RHEUMATOID ARTHRITIS INDUCED BY ALPHA-
\end{tabular} INTERFERON THERAPY: A RARE CASE PRESENTATION}

M. Yasmine ${ }^{1}$, H. Ajlani ${ }^{1}$, S. Boussaud ${ }^{1}$, S. Jammali ${ }^{1}$, H. Sehli ${ }^{1}$, E. Choeur ${ }^{1}$, S. Rekik ${ }^{1}$, M. El Euch ${ }^{1}{ }^{1}$ Rabta, Tunis, Tunisia

Background: Interferon-a (IFN-a) is known for its antiviral and antiproliferative effects, used mainly for the treatment of chronic hepatitis $C$ infection [1]. Immunomodulatory effects have been reported in patients treated with IFN-a, including hematological, immunological, rheumatological and dermatological disorders [2]. In fact, IFN-a may lead to the induction or exacerbation of autoimmune diseases such as psoriasis, systemic lupus erythematosus, and rarely rheumatoid arthritis (RA)

Objectives: We report the case of a Caucasian who developed anticyclic citrullinated peptide antibody (anti-CCP)-positive RA following treatment of chronic hepatitis $\mathrm{C}$ infection with pegylated IFN- $a 2 \mathrm{a}$.

Methods: A 57-year-old women was diagnosed of chronic hepatitis $\mathrm{C}$ infection after detection of abnormal liver function. She has a genotype $\mathrm{lb}$ with a high viral load: RNA was $100,000 \mathrm{UI} / \mathrm{ml}$. Liver histology showed advanced fibrosis and portal fibrosis (A3 F4 according to metavir score). A history of blood transfusion was found. The patient was placed on a 24-week course of PEGylated -IFN-a2a $180 \mu \mathrm{g}$ weekly and a $1000 \mathrm{mg}$ daily dose of ribavirin. After 
two months of antiviral treatment, she developed symmetrical polyarthritis, with pain and edema in the wrists, elbows, shoulders and metacarpophalangeal joints, associated with prolonged morning stiffness. The musculoskeletal examination was notable for active synovitis of the proximal phalangeal joints, metacarpophalangeal joints, wrists, elbows. Distal interphalangeal joints were spared. She had no musculoskeletal symptoms prior to antiviral therapy. Review of systems was otherwise unremarkable. X-ray showed no remarkable findings. Ultrasonography of the hands revealed diffuse synovitis as well as tenosynovitis of the ulnar extensor tendons in both wrists. Laboratory results revealed a normal C-reactive protein, elevated liver enzymes: ALAT (alanin-aminotransferase) 119,ASAT (aspartat-aminotransferase) 66, Gamma-GT 203 and undetectable cryoglobulins. Anti-CCP was $21 \mathrm{IU} / \mathrm{ml}$ (negative $<20$ $\mathrm{IU} / \mathrm{ml}$ ), antinuclear antibodies were positive 1280 (negative $<160$ ), rheumatoid factor was 192 (normal < $30 \mathrm{lU} / \mathrm{ml})$.

A diagnosis of rheumatoid arthritis (RA) was made on the basis of clinical and ultra-sonographic evidence as well as Rheumatoid Factor (RF) and anti-cyclic citrullinated peptide (anti-CCP) antibody positivity. Moreover, an autoimmune thyroiditis was found that evolved into hypothyroidism treated with thyroxine. Results: The patient developed a sustained virological response as evidenced by persistent undetectable HCV RNA and normal aminotransferase activities. Upon completion of a 12-week course of antiviral therapy, The rheumatoid syndrome disappeared after cessation of IFN therapy. By that time, antinuclear antibodies were in a titre of $1 / 180$, rheumatoid factor and Anti-CCP were negative.

Conclusion: The present case suggests that biological agents, affecting the cytokine network, may work as triggering factors for the development of RA in previously predisposed individuals. Screening for RF and anti-CCP may be considered before treating with IFN. In addition, a close surveillance for the occurrence of autoimmune phenomena during and after treatment should be worthy, for early diagnosis and adequate clinical management.

References:

[1] DARICE YANG, DANIEL ARKFELD and TSE-LING FONG Treatment for Chronic Hepatitis C Infection. Development of Anti-CCP-positive Rheumatoid Arthritis Following Pegylated Interferon-alpha. J Rheumatol 2010;37;1777Volume 37, no. 8. 2a

[2] Okanoue T, Sakamoto S, Itoh Y, Minami M, Yasui K, Sakamoto M, et al. Side effects of high-dose interferon therapy for chronic hepatitis C. J Hepatol 1996;25:283-91.

Disclosure of Interests: None declared

DOI: 10.1136/annrheumdis-2020-eular.2286

\section{THURSDAY, 04 JUNE 2020}

\section{HPR Measuring health (development and measure- ment properties of PROs, tests, devices)}

\section{THU0607-HPR COMPARISON OF THE PATIENT REPORTED PHYSICAL ACTIVITY LEVEL ACCORDING TO KINESIOPHOBIA PRESENCE IN PATIENTS WITH AXIAL SPONDYLOARTHRITIS}

D. Bayraktar ${ }^{1}$, D. Ozer Kaya ${ }^{1}$, S. Gucenmez ${ }^{2}$, E. Durak Ediboglu ${ }^{3}$, G. Kabadayi ${ }^{3}$, S. Akar ${ }^{3}{ }^{1}$ Izmir Katip Celebi University, Faculty of Health Sciences, Department of Physiotherapy and Rehabilitation, Izmir, Turkey; ${ }^{2}$ Izmir Katip Celebi University, Ataturk Education and Research Hospital, Department of Rheumatology, Izmir, Turkey; ${ }^{3}$ Izmir Katip Celebi University, Faculty of Medicine, Department of Internal Medicine, Division of Rheumatology, Izmir, Turkey

Background: Many factors such as poor functional or emotional status might play a role in participating physical activity for people with rheumatic diseases. There is a lack for evidence regarding to the effect of kinesiophobia presence on the physical activity levels of axSpA patients.

Objectives: The primary objective was to compare the patient reported physical activity levels in axSpA patients with kinesiophobia and those without. Evaluating disease related and physical characteristics, quality of life and emotional status according to presence of kinesiophobia were also aimed.

Methods: One-hundred forty-eight consecutive axSpA patient were allocated to Kinesiophobia+ group (n: 90, 66\% males) or Kinesiophobia- group (n: 58, 64\% males). The presence of kinesiophobia was defined as having a score of $>37$ in Tampa Scale for Kinesiophobia. All patients were evaluated regarding to physical characteristics (age, body-mass index), functional status (Bath Ankylosing Spondylitis Functional Index), disease activity (Bath Ankylosing Spondylitis Disease Activity Index), spinal mobility (Bath Ankylosing Spondylitis Metrology Index), patient reported physical activity (International Physical Activity Questionnaire Short Form), emotional status (Hospital Anxiety and Depression Scale), and quality of life (Assessment of SpondyloArthritis International Society Health Index).
Results: Physical characteristics and spinal mobility were similar in patients with and without kinesiophobia ( $p>0.05$, Table). Disease activity, function, quality of life, depression and anxiety scores were poorer in Kinesiophobia+ group compared to Kinesiophobia- group ( $p<0.05$, Table). Patient reported physical activity level was found to be lower in patients with kinesiophobia $(p<0.05$, Table).

Table. Comparison of groups according to kinesiophobia presence

\begin{tabular}{|c|c|c|c|}
\hline & $\begin{array}{c}\text { Kinesiophobia+ Group } \\
\text { (n: 90) } \\
\text { Median (IQR 25/75) }\end{array}$ & $\begin{array}{c}\text { Kinesiophobia- Group } \\
\text { (n: 58) } \\
\text { Median (IQR 25/75) }\end{array}$ & $\mathbf{p}^{*}$ \\
\hline \multicolumn{4}{|l|}{ Physical Characteristics } \\
\hline Age (years) & $41.0(37.0 / 52.0)$ & $44.0(36.0 / 53.0)$ & 0.850 \\
\hline Body-Mass Index $\left(\mathrm{kg} / \mathrm{m}^{2}\right)$ & $26.8(24.6 / 30.1)$ & $26.0(22.7 / 29.0)$ & 0.364 \\
\hline \multicolumn{4}{|c|}{ Disease Related Characteristics } \\
\hline BASDAI (score) & $2.7(1.3 / 4.6)$ & $1.8(0.6 / 3.5)$ & 0.036 \\
\hline BASMI (score) & $3.3(1.9 / 5.0)$ & $2.7(1.6 / 3.5)$ & 0.114 \\
\hline BASFI (score) & $2.5(1.2 / 4.4)$ & $1.2(0.5 / 2.7)$ & 0.003 \\
\hline \multicolumn{4}{|c|}{ Patient Reported Physical Activity Level } \\
\hline IPAQ (MET/week) & $505.5(169.0 / 1653.0)$ & $858.0(330.0 / 2772.0)$ & 0.042 \\
\hline \multicolumn{4}{|l|}{ Quality of Life } \\
\hline ASAS-HI (score) & $7.0(5.0 / 9.1)$ & $4.0(2.0 / 7.9)$ & 0.001 \\
\hline \multicolumn{4}{|l|}{ Emotional Status } \\
\hline HAD Anxiety (score) & $7.0(4.0 / 9.0)$ & $5.0(3.0 / 8.0)$ & 0.006 \\
\hline HAD Depression (score) & $7.0(4.0 / 10.0)$ & $3.0(1.0 / 7.0)$ & 0.001 \\
\hline \multicolumn{4}{|l|}{ Fear of Movement } \\
\hline TAMPA (score) & $41.0(39.0 / 44.0)$ & $34.0(31.0 / 36.0)$ & $\mathrm{p}<0.001$ \\
\hline
\end{tabular}

*Mann-Whitney U Test, IQR 25/75: Interquartile range 25/75, BASDAI: Bath Ankylosing Spondylitis Disease Activity Index, BASMI: Bath Ankylosing Spondylitis Metrology Index BASFI: Bath Ankylosing Spondylitis Functional Index, IPAQ: International Physical Activity Questionnaire Short Form, HAD: Hospital Anxiety and Depression Scale, ASAS-HI: Assessment of SpondyloArthritis International Society Health Index, TAMPA: The Tampa Scale for Kinesiophobia, $\mathrm{p}<0.05$

Conclusion: It seems that the presence of kinesiophobia may have a negative impact on patient reported physical activity level, and disease related parameters. However, it is also possible that kinesiophobia might occur as result of poor disease activity or disability. Strategies such as patient education should be included in axSpA management for preventing kinesiophobia development or improving kinesiophobia related consequences.

Disclosure of Interests: None declared

DOI: 10.1136/annrheumdis-2020-eular.4130

\section{THU0608-HPR VALIDITY OF SIX MINUTE STEPPER TEST IN EVALUATION OF FUNCTIONAL EXERCISE CAPACITY IN PATIENTS WITH ANKYLOSING SPONDYLITIS}

S. Bayram ${ }^{1}$, N. G. Tore ${ }^{1}$, F. Sari ${ }^{1}$, D. C. Saraç ${ }^{1}$, G. Gülsün ${ }^{1}$, R. Bilici ${ }^{2}$, A. Tufan ${ }^{2}$, D. Oskay ${ }^{1}{ }^{1}$ Gazi University Faculty of Health Sciences, Department of Physiotherapy and Rehabilitation, Ankara, Turkey; ${ }^{2}$ Gazi University Faculty of Medicine, Department of Internal MedicineRheumatology, Ankara, Turkey

Background: In most patients with ankylosing spondylitis (AS), exercise capacity decreases due to pulmonary dysfunction, chest wall restriction and peripheral muscle weakness. The six-minute walk test (6MWT) is a validated simple field, hence frequently used to evaluate exercise capacity. However, 6MWT has some limitations, especially the fact that it requires a corridor of at least 30 meters long to perform this test which can limit its use in some centers. Shorter corridors force patients to turn more frequently, slowing down the pace of walking that reduces potential walking distance. To overcome technical and spatial limitations, 6-minute stepper test (6MST) has been proposed to evaluate exercise capacity. In the literature 6MST has been suggested for a variety of diseases. Since, it requires only a limited amount of space and equipment and is feasible, easy to perform, well tolerated.

Objectives: In the literature, there is no study in which 6MST is used to evaluate exercise capacities of patients with AS. Therefore, the aim of this study was to evaluate validity of 6MST in AS population in comparison to 6MWT.

Methods: 6MWT and 6MST were performed in 51 patients with AS $(52.26 \pm 13.33$ years, 30F/21M). Demographic and clinical characteristics were recorded. Functional exercise capacity was evaluated using 6MWT and 6MST. The total distance of 6MWT was compared to the total number of steps of 6MST. Before, during and after 6MWT and 6MST, heart rate $(\mathrm{HR})$, oxygen saturation $\left(\mathrm{SpO}_{2}\right)$, breathing frequency (BF), blood pressure (BP), dyspnea and fatigue were assessed using modified Borg scale.

Results: The number of steps on the 6MST was significantly correlated with the distance of the 6MWT $(r=0.61, p<0.0001)$. Dyspnea $(p=0.04)$ and leg fatigue $(p<0.0001)$ was significantly higher in $6 \mathrm{MST}$ than in $6 \mathrm{MWT}$. HR, SpO $, \mathrm{BF}, \mathrm{BP}$ and fatigue were similar in both 6MST and 6MWT. 